\title{
Exercise capacity in relation to autoantibodies in systemic sclerosis patients
}

\author{
Fujiko Someya $\cdot$ Naoki Mugii $\cdot$ Tetsutarou Yahata $\cdot$ \\ Takao Nakagawa
}

Received: 15 March 2012/Accepted: 21 October 2012/Published online: 5 November 2012

(C) The Author(s) 2012. This article is published with open access at Springerlink.com

\begin{abstract}
Autoantibodies have been detected in systemic sclerosis patients, and typical clinical features regarding organ involvement by each autoantibody have been reported. To reveal differences in exercise intolerance in patients with either anti-topoisomerase-I or anti-centromere antibodies, 53 systemic sclerosis patients were investigated retrospectively. A 6-min walking distance showed no significant differences $(P=0.090)$ between autoantibodies, while exercise-induced hypoxia during the 6-min walking test was significant in subjects with the anti-topoisomerase-I antibody $(P=0.033)$. The percent predicted of vital capacity, the diffusion capacity of the lung for carbon monoxide, and the modified Rodnan skin score were affected more in subjects with the anti-topoisomerase-I antibody than the anti-centromere antibody. The main parameter affecting the 6-min walking distance was the percent predicted of vital capacity for each autoantibody, and there was a significant positive relationship for all subjects $\left(R^{2}=0.30, P<0.0001\right)$. Exercise-induced hypoxia was also shown in the more affected subjects in the percent predicted of vital capacity and the diffusion capacity of the lung for carbon monoxide. Lung parameters were suggested to be more important factors determining exercise intolerance and induced hypoxia than detected autoantibodies.
\end{abstract}

Keywords Systemic sclerosis - 6-Min walking test . Autoantibody $\cdot$ Lung function · Oxygen saturation

F. Someya $(\square) \cdot$ T. Nakagawa

School of Health Sciences, Kanazawa University,

Kodatsuno 5-11-80, Kanazawa 920-0942, Japan

e-mail: fujiko@mhs.mp.kanazawa-u.ac.jp

N. Mugii · T. Yahata

Division of Rehabilitation, Kanazawa University Hospital,

Takaramachi 13-1, Kanazawa 920-8640, Japan

\section{Introduction}

Systemic sclerosis (SSc) is classified into two subsets, diffuse cutaneous SSc and limited cutaneous SSc, according to the extent of skin lesions. Some autoantibodies in sera found in SSc patients include anti-topoisomerase-I and anti-centromere antibodies [1-3]. The anti-topoisomerase-I antibody likely causes diffuse cutaneous SSc and a significant increased risk of lung and heart involvement. In contrast, the anti-centromere antibody causes limited cutaneous SSc and less organ involvement.

Exercise intolerance was also examined in SSc patients regarding organ dysfunction [4], and interstitial lung disease was suggested to be important in predicting mortality [5]. However, there are few studies regarding the role of autoantibodies on exercise capacity. The anti-topoisomerase-I antibody (anti-Sel-70 autoantibody) was reported to be a factor determining oxygen desaturation during the 6-min walking test (6MWT), where compared autoantibodies in the analysis were unclear [6].

The aim of this study is to reveal the role of autoantibodies on exercise capacity. Since possible organ involvement by each autoantibody is becoming clear, it must be established whether exercise intolerance is typically found in SSc with the anti-topoisomerase-I antibody or is caused by organ dysfunction despite autoantibody specificity.

\section{Methods}

Fifty-three consecutive SSc patients with either anti-topoisomerase-I or anti-centromere antibodies who could perform the 6MWT were assigned to this study retrospectively (Table 1). Patients were referred as part of their routine 
Table 1 Characteristics of subjects

Values are mean $\pm \mathrm{SD}$

NA not available, MRSS modified Rodnan skin score, 6MWT 6-minute walking test

\begin{tabular}{llll}
\hline Parameters & Anti-topoisomerase-I antibody & Anti-centromere antibody & $P$ \\
\hline Sex (f/m) & $32 / 5$ & $15 / 1$ & 0.65 \\
Age (years) & $54.3 \pm 12.3$ & $63.0 \pm 8.9$ & 0.0062 \\
Duration of disease (years) & $5.0 \pm 5.1$ & $10.9 \pm 11.1$ & 0.057 \\
Subset (diffuse/limited) & $30 / 7$ & $0 / 16$ & NA \\
Vital capacity (\% pred) & $85.5 \pm 22.9$ & $110.6 \pm 20.3$ & 0.0004 \\
D & $53.9 \pm 16.8$ & $68.2 \pm 19.9$ & 0.019 \\
MRSS & $14.6 \pm 9.9$ & $3.6 \pm 4.3$ & $<0.0001$ \\
6MWT distance $(\%$ pred) & $85.7 \pm 15.7$ & $95.1 \pm 18.8$ & 0.090 \\
Induced hypoxia/negative & $20 / 17$ & $3 / 13$ & 0.033 \\
\hline
\end{tabular}

evaluation for treatment. Evaluations were performed during their first visit to our facility from 2007 to 2011. The study was approved by the Ethics Committee of our facility. Oxygen saturation $\left(\mathrm{SpO}_{2}\right)$ was monitored during the 6MWT. The $\mathrm{SpO}_{2}$ value was sufficiently high in all subjects $(>95 \%)$ at rest, while a decrease from baseline $\geq 4 \%$ after the 6MWT was defined as exercise-induced hypoxia. Percent predicted of vital capacity (VC) and the diffusion capacity of the lung for carbon monoxide $\left(\mathrm{D}_{\mathrm{LCO}}\right)$ from spirometry were collected as pulmonary parameters. Skin involvement was evaluated by the modified Rodnan skin score.

\section{Statistics}

Walking distance was calculated as a percent of predicted values by the Enright formula [7] taking gender, age, height, and weight into consideration. Differences in evaluation values between the two autoantibodies were compared using the $t$ test. The chi-square test was used for the comparison of sex distribution, subset of disease, and exercise-induced hypoxia. Simple linear regression between the 6MWT distance and parameter values was performed for each autoantibody. Statistical analyses were performed with JMP8.0 (SAS Institute, Cary, NC, USA). In all analyses, $P<0.05$ was taken to indicate significance.

\section{Results}

The anti-topoisomerase-I antibody was detected in 37 of 53 subjects and 30 of them were diagnosed with diffuse cutaneous SSc, whereas all 16 subjects with the anti-centromere antibody had limited cutaneous SSc (Table 1). There was no difference in sex distribution between the autoantibodies, though age was significantly higher and duration of disease after the onset of Raynaud's phenomenon tended to be longer in subjects with the anti-centromere antibody. There was no significance in the 6MWT distance between
Table 2 Simple linear regression analysis between the 6MWT distance and parameters

\begin{tabular}{|c|c|c|c|c|}
\hline \multirow[t]{2}{*}{ Parameters } & \multicolumn{2}{|c|}{$\begin{array}{l}\text { Anti-topoisomerase-I } \\
\text { antibody }\end{array}$} & \multicolumn{2}{|c|}{$\begin{array}{l}\text { Anti-centromere } \\
\text { antibody }\end{array}$} \\
\hline & $R^{2}$ & $P$ & $R^{2}$ & $P$ \\
\hline Vital capacity (\% pred) & 0.22 & 0.0034 & 0.33 & 0.020 \\
\hline $\mathrm{D}_{\mathrm{LCO}}(\%$ pred $)$ & 0.016 & 0.46 & 0.13 & 0.18 \\
\hline MRSS & 0.086 & 0.079 & 0.044 & 0.44 \\
\hline
\end{tabular}

MRSS the modified Rodnan skin score

autoantibodies $(P=0.090)$, while hypoxia induced by the 6MWT was significant in subjects with the anti-topoisomerase-I antibody $(P=0.033)$. Moreover, percent predicted of VC, $\mathrm{D}_{\mathrm{LCO}}$, and the modified Rodnan skin score were more affected in subjects with the anti-topoisomeraseI antibody than the anti-centromere antibody.

The only parameter affecting the 6MWT distance was percent predicted of VC for each autoantibody (Table 2). The regression line between the 6MWT distance and percent predicted of VC for all subjects represented a positive relationship $\left(R^{2}=0.30, P<0.0001\right)$ (Fig. 1). Induced hypoxia was shown in the more affected subjects in percent predicted of $\mathrm{VC}$ and $\mathrm{D}_{\mathrm{LCO}}$, but not in age, duration of disease, or the 6MWT distance (Table 3).

\section{Discussion}

In this study, as supported by previous studies [1, 3], lung and skin involvement was found in SSc with the antitopoisomerase-I antibody more than that with the anticentromere antibody. Moreover, the tendency of a longer duration from the onset of disease without severe organ dysfunction in subjects with the anti-centromere antibody was suggested by the rapid progress in lung involvement by the anti-topoisomerase-I antibody [8].

The main aim of this study was to define the limiting factors of exercise capacity. The distance of the 6MWT 


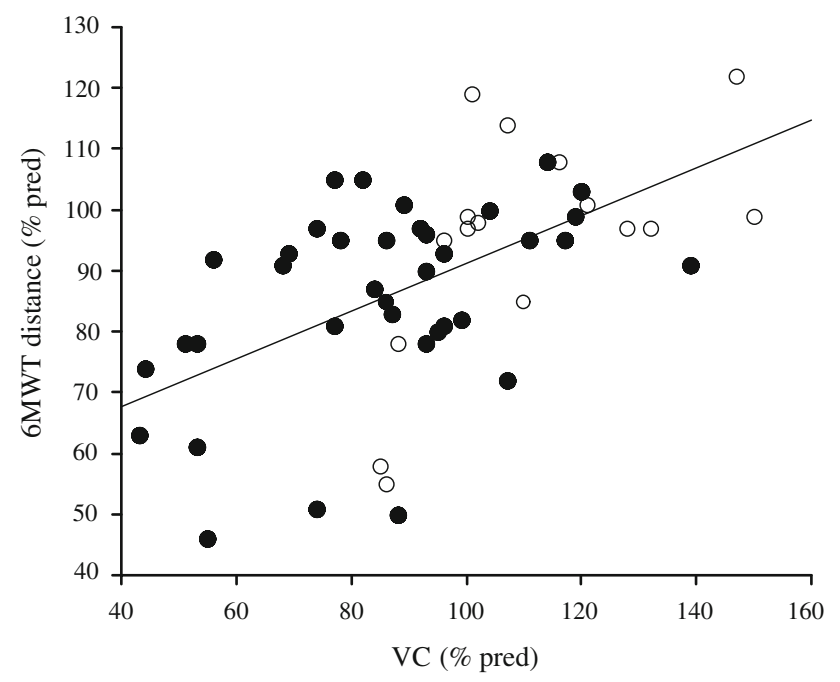

Fig. 1 Relationship of percent predicted of vital capacity to the 6MWT distance. Closed circles represent the anti-topoisomerase-I antibody and open circles represent the anti-centromere antibody. The regression line is the $6 \mathrm{MWT}$ distance $(\%$ pred $)=53.8+0.37 * \mathrm{VC}$ (\% pred) $\left(R^{2}=0.30, P<0.0001\right)$

Table 3 Effects of parameters on exercise-induced hypoxia

\begin{tabular}{lllc}
\hline Parameters & $\begin{array}{l}\text { Induced } \\
\text { hypoxia }\end{array}$ & $\begin{array}{l}\text { Negative } \\
\text { response }\end{array}$ & $P$ \\
\hline Age (years) & $57.3 \pm 10.7$ & $56.7 \pm 13.0$ & 0.85 \\
Duration of disease (years) & $6.0 \pm 4.5$ & $7.3 \pm 9.7$ & 0.51 \\
Subset (diffuse/limited) & $17 / 6$ & $13 / 17$ & 0.049 \\
Vital capacity (\% pred) & $78.3 \pm 21.2$ & $104.3 \pm 21.5$ & $<0.0001$ \\
D LCO (\% pred) & $45.4 \pm 13.2$ & $68.1 \pm 16.4$ & $<0.0001$ \\
MRSS & $12.7 \pm 9.8$ & $10.3 \pm 10.1$ & 0.38 \\
6MWT distance (\% pred) & $86.4 \pm 19.4$ & $90.1 \pm 15.2$ & 0.45 \\
\hline
\end{tabular}

Values are mean $\pm \mathrm{SD}$

MRSS the modified Rodnan skin score, 6MWT 6-minute walking test

tended to be shorter in SSc with the anti-topoisomerase-I antibody than that with the anti-centromere antibody, but there was no significant difference despite distinguishable lung and skin involvement. However, simple linear regression analysis showed a clear relationship between the 6MWT distance and percent predicted of VC. These results suggested exercise intolerance was mainly caused by lung dysfunction, which was also shown in subjects with the anti-centromere antibody.

Exercise-induced hypoxia was more common in SSc with the anti-topoisomerase-I antibody than that with the anti-centromere antibody [6]. Since there were only three subjects with the anti-centromere antibody showing induced hypoxia, it was difficult to detect the affecting factors on induced hypoxia divided by each autoantibody. Lung involvement was significantly severe in subjects with induced hypoxia; however, skin involvement and/or exercise capacity did not affect oxygen saturation. Therefore, there remained the possibility that induced hypoxia was also caused by lung involvement and not by autoantibodies per se.

Other autoantibodies include anti-RNA polymerase, anti-U1-RNP, and anti-U3-RNP antibodies. As the antiU1-RNP antibody is known to cause isolated pulmonary arterial hypertension [9], there is the possibility that a different relationship could exist between exercise capacity and examined parameters. This is because exercise capacity could also be reduced by pulmonary arterial hypertension $[6,10]$. The distribution of autoantibodies in SSc has regional variety [3], and there were only two patients with the anti-U1-RNP antibody in this study. We excluded such a small number of cases and examined only two major autoantibodies. In the 53 subjects in this study, there was no relationship between the 6MWT distance and right ventricular systolic pressure $\left(R^{2}=0.0013\right.$, $P=0.79$ ), which may be the result of comparatively low pulmonary arterial pressures in these subjects.

Detection of autoantibodies would be beneficial to SSc patients for predictive prognosis concerning organ involvement. Even though the anti-centromere antibody has less of an effect on organs than that of the anti-topoisomerase-I antibody, organ involvement could not be avoided in disease of a long duration. Lung parameters were suggested to be important determinants of exercise intolerance and induced hypoxia in spite of whichever autoantibody was positive. In conclusion, careful examination of organ involvement is necessary regarding exercise capacity even after detection of autoantibodies.

Acknowledgments This study was supported by a Grant-in-Aid for Scientific Research (C) (21500466).

Conflict of interest No conflicts of interest exist.

Open Access This article is distributed under the terms of the Creative Commons Attribution License which permits any use, distribution, and reproduction in any medium, provided the original author(s) and the source are credited.

\section{References}

1. Hashimoto A, Endo H, Kondo H, Hirohata S (2011) Clinical features of 405 Japanese patients with systemic sclerosis. Mod Rheumatol 22:272-279

2. Cuomo G, Santoriello C, Polverino F, Ruocco L, Valentini G, Polverino M (2010) Impaired exercise performance in systemic sclerosis and its clinical correlations. Scand J Rheumatol 39:330-335. doi:10.3109/03009740903555358

3. Ho KH, Reveille JD (2003) The clinical relevance of autoantibodies in scleroderma. Arthr Res Ther 5:80-93. doi:10.1186/ar628

4. Garin MC, Highland KB, Silver RM, Strange C (2009) Limitations to the 6-minute walk test in interstitial lung disease and 
pulmonary hypertension in scleroderma. J Rheumatol 36:330-336. doi: $10.3899 /$ jrheum.080447

5. Goh NSL, Desal SR, Veeraraghavan S et al (2008) Interstitial lung disease in systemic sclerosis: a simple staging system. Am J Respir Crit Care Med 177:1248-1254. doi:10.1164/rccm.200706$8770 \mathrm{C}$

6. Villalba WO, Sampaio-Barros PD, Pereira MC et al (2007) Sixminute walk test for the evaluation of pulmonary disease severity in scleroderma patients. Chest 131:217-222. doi:10.1378/chest. 06-0630

7. Enright PL, Sherrill DL (1998) Reference equations for the sixminute walk in healthy adults. Am J Respir Crit Care Med 158: 1384-1387
8. Hasegawa M, Asano Y, Endo H et al (2012) Investigation of prognostic factors for skin sclerosis and lung function in Japanese patients with early systemic sclerosis: a multicentre prospective observational study. Rheumatology 51:129-133. doi:10.1093/ rheumatology/ker333

9. Hamaguchi Y (2010) Autoantibody profiles in systemic sclerosis: predictive value for clinical evaluation and prognosis. J Dermatol 37:42-53. doi:10.1111/j.1346-8138.2009.00762.x

10. Alkotob ML, Soltani P, Sheatt MA et al (2006) Reduced exercise capacity and stress-induced pulmonary hypertension in patients with Scleroderma. Chest 130:176-181. doi:10.1378/chest.130.1.176 